\title{
Detecting the Number of Transmit Antennas with Unauthorized or Cognitive Receivers in MIMO Systems
}

\author{
Oren Somekh*, Osvaldo Simeone*, Yeheskel Bar-Ness*, and Wei Su ${ }^{\dagger}$ \\ * CWCSPR, Department of Electrical and Computer Engineering, NJIT, Newark, NJ 07102, USA \\ $\dagger$ U.S. Army RDECOM CERDEC, Fort Monmouth, NJ 07703 USA
}

\begin{abstract}
In this work we consider one of the challenges facing unauthorized receivers and cognitive radios since the appearance of MIMO system, which is detecting the number of transmit antennas. To achieves this goal, we present a detector based on objective information theoretic criteria (the celebrated AIC and MDL estimators). Numerical results derived for an ideal BLAST-like transmission demonstrate good performance already for mild SNR conditions and majority decision based on rather few independent measurements.
\end{abstract}

\section{INTRODUCTION}

The great potential provided by the use of multiple input multiple output (MIMO) arrays [1][2], encouraged intensive research efforts in the fields of information theory and communications in recent years. This effort led to the design and implementation of new commercial communication systems which include MIMO arrays, such as BLAST, WiFi (802.11n), WiMax (802.16), and 4G cellular systems. In addition, many proprietary MIMO based systems which do not follow any commercial standard are also being used for military communication. This new scenario introduces many new challenges for both cognitive radios and surveillance systems.

In this work we focus on one of these challenges, which is detecting the number of transmit antennas of a targeted transmission. Towards this end, we utilize objective information theoretic criteria (AIC and MDL) which were successfully applied to estimate the number of (possibly correlated) single antenna emitters [3]. We present numerical results of these estimators for fully synchronized BLAST like transmissions and Rayleigh fading channels. Numerical results presented for this idealized setup show that the AIC/MDL estimators provides a robust detector for detecting the number of transmit antennas, based on the eigenvalues of the sample covariance matrix. The coherence time of the channel (interpreted as the measurement length) is a critical system parameter where for large measurement lengths, the detection probability in general increases with the receive array size. It is also verified that for a given set of parameters, there is a crucial value of SNR, above which "hard" combining of multiple independent measurements quickly brings the final detection probability to 1. Finally we point out several possible extensions to the considered basic ideal setup which are currently under investigation; namely, the impact of: coding, channel characterization, and imperfect reception, on the system performance.

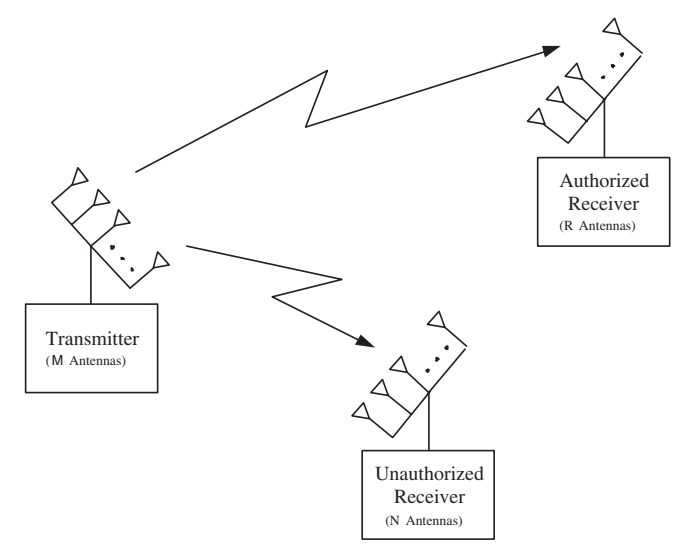

Fig. 1. System setup

\section{Problem Statement}

We consider an isolated setup (see Figure 1), in which a single multi-antenna terminal is communicating with an authorized multi-antenna terminal. A multi-antenna unauthorized (or cognitive) terminal is intercepting the transmission whose goal is to detect the number of transmit antennas.

Underlying assumptions:

- Transmitter:

- Uncorrelated $M$ transmit antennas.

- The i.i.d. zero-mean complex Gaussian input vector is independent of the channel.

- Unauthorized/Cognitive terminal:

- Uncorrelated $N$ receive antennas $(N>M)$.

- Perfect synchronization (timing, carrier frequency, and carrier phase)

- No channel state information (CSI).

- Channel:

- I.i.d block flat fading channel.

- Continuous fading distribution.

- Coherence time $T_{\mathrm{c}}>T$ (in symbols).

- The i.i.d. zero-mean complex Gaussian additive noise vector, is independent of the channel transfer matrix and the transmitted signals.

Accounting for the underling assumptions, a baseband representation of the received $N \times 1$ vector in the $i$ 'th time index 
for an arbitrary fading block is given by

$$
\boldsymbol{y}_{i}=\boldsymbol{H} \boldsymbol{x}_{i}+\boldsymbol{n}_{i} \quad ; \quad i=0,1, \ldots, T-1,
$$

where $\boldsymbol{x}_{i}$ is the complex Gaussian transmitted vector $\boldsymbol{x}_{i} \sim$ $\mathcal{C N}\left(\mathbf{0}, \frac{P}{M} \boldsymbol{I}_{M}\right), \boldsymbol{n}_{i}$ is the complex Gaussian additive noise $N \times 1$ vector $\boldsymbol{n}_{i} \sim \mathcal{C N}\left(\mathbf{0}, \sigma^{2} \boldsymbol{I}_{N}\right)$, and $\boldsymbol{H}$ is the channel transfer $N \times M$ matrix between the transmitter and the interceptor. Since continuous fading distributions are assumed, $\boldsymbol{H}$ is almost surely full ranked.

\section{Detecting the Number of Transmit Antennas}

\section{A. Intuition}

Since the noise vector is zero mean and independent of the transmitted signals and the channel, the conditioned covariance matrix of the received signal vector is given by

$$
\boldsymbol{R}=E\left\{\boldsymbol{y} \boldsymbol{y}^{\dagger} \mid \boldsymbol{H}\right\}=\boldsymbol{\Psi}+\sigma^{2} \boldsymbol{I}_{N},
$$

where

$$
\boldsymbol{\Psi}=\boldsymbol{H} \boldsymbol{H}^{\dagger}
$$

Since $\boldsymbol{H}$ is full ranked, it follows that the rank of $\Psi$ is $\min (M, N)=M$, which is the number of transmit antennas. Hence, the smallest $(N-M)$ ordered eigenvalues of $\boldsymbol{R}$ equal $\sigma^{2}$, i.e. $\lambda_{M+1}=\lambda_{M+2}=\cdots=\lambda_{N}=\sigma^{2}$. It is concluded that given the covariance matrix $\boldsymbol{R}$ the number of transmit antennas can be determined from the cardinality of the smallest eigenvalues of $\boldsymbol{R}$. Since $\boldsymbol{R}$ is unknown to the interceptor and it is estimated from a finite set of noisy samples vectors, the resulting smallest $(N-M)$ eigenvalues are different in probability one, making it difficult to determined the number of transmit antenna merely by "observing" the eigenvalues.

\section{B. Subjective Criteria}

A more sophisticated approach to the problem developed by Bartlett [4] and Lawley [5], is based on a a sequence of hypothesis tests. For each hypothesis, the likelihood ratio statistics is computed and compared to a threshold. The hypothesis accepted is the first one for which the threshold is crossed. The problem associated with this approach is the subjective judgement needed in the selection of the threshold levels for the different tests.

\section{Information Theoretic Criteria}

Expression (1) can be rewritten as

$$
\boldsymbol{y}_{i}=\sum_{m=1}^{M}[\boldsymbol{H}]_{m}\left[\boldsymbol{x}_{i}\right]_{m}+\boldsymbol{n}_{i},
$$

where $[\boldsymbol{H}]_{m}$ is the $m$ 'th column of $\boldsymbol{H}$, and $\left[\boldsymbol{x}_{i}\right]_{m}$ is the $m$ 'th entry of the symbols vector $\boldsymbol{x}_{i}$. Examining (4) and accounting for the underling assumptions, it is concluded that the problem of determining the number of transmit antennas in its simplest form, is equivalent to determining the number of single antenna sources considered by Wax and Kailath in [3]. The latter paper takes a different approach than the approach of [4][5], and the detection problem is interpreted as a model selection problem. Next, information theoretic criteria for model selection, introduced by Akaike [6][7], and by Schwartz [8] and Rissanen [9], are applied, and the number of transmit antennas is determined as the value for which the AIC or minimum descriptor length (MDL) criteria is minimized.

Following [3], the form of AIC for this problem is given by

$$
\begin{aligned}
& \operatorname{AIC}(m)=-2(N-m) T \log \left(\frac{\prod_{i=m+1}^{N} l_{i}^{1 /(N-m)}}{\frac{1}{N-m} \sum_{i=m+1}^{N} l_{i}}\right) \\
& +2 m(2 N-m) \text {, }
\end{aligned}
$$

while the MDL criterion is given by

$$
\begin{aligned}
\operatorname{MDL}(m)=-(N-m) T \log & \left(\frac{\prod_{i=m+1}^{N} l_{i}^{1 /(N-m)}}{\frac{1}{N-m} \sum_{i=m+1}^{N} l_{i}}\right) \\
& +\frac{1}{2} m(2 N-m) \log T,
\end{aligned}
$$

where $l_{1}>l_{2} \cdots>l_{N}$ are the ordered eigenvalues of the sample covariance matrix $\hat{\boldsymbol{R}}$ defined by

$$
\hat{\boldsymbol{R}} \triangleq \frac{1}{T} \sum_{i=0}^{T-1} \boldsymbol{y}_{i} \boldsymbol{y}_{i}^{\dagger},
$$

and $T$ is the the number of samples vectors available for the empirical second order statistics calculations. It is noted that the AIC and the MDL criteria are related according to the following

$$
\mathrm{AIC}(m)=2 \operatorname{MDL}(m)+m(2 N-m)(2-\log T) .
$$

The estimated number of transmit antennas $\hat{M}$ is determined as the value of $m=\{0,1, \cdots, N-1\}$ for which either the AIC or MDL is minimized. i.e. for the MDL we have

$$
\hat{M}=\underset{m=0,1, \ldots, N-1}{\operatorname{argmin}} \operatorname{MDL}(m) .
$$

It is shown in [3] that the MDL yields a consistent estimate ${ }^{1}$, while the AIC yields an inconsistent estimate that tends, asymptotically with the number of samples, to overestimate the number of transmit antennas.

\section{Multiple Independent Measurements}

Since the Frobenius norm of the sample matrix $\hat{\boldsymbol{R}}$ converge almost surely to the covariance matrix $\boldsymbol{R}$ with $T$, it is evident that the performance of the MDL/AIC estimators improve for increasing number of samples vectors. However, in real scenarios the channel coherence time $T_{\mathrm{c}}$ is finite, and its value depends on the system parameters and the dynamics of the environment. On the other hand, it is reasonable to assume that the communication session between the transmitter and the receiver may typically last much longer than $T_{\mathrm{c}}$, and the interceptor may conduct many independent measurements, each of $T$ samples. Then the $L$ interim measurements may be combined in a "hard" or "soft" manner to produce the final estimation. It is noted that since the channel is assumed block independent and that the measurement periods are

\footnotetext{
${ }^{1} \mathrm{~A}$ consistent estimator is an estimator that converges in probability to the quantity being estimated as the sample size grows.
} 
synchronized with the channel blocks transitions, the detector interim outputs are i.i.d.

A "hard" combiner detector makes its final estimation $\hat{M}$ based on a majority decision. In case the majority decision is not unique, the detector randomly picks its decision among the multiple choices. To assess the performance of a "hard" combiner detector, let us define

$$
\begin{gathered}
P_{N}^{M}(n) \triangleq \operatorname{Pr}\left(n=\min _{m} \operatorname{MDL}(m) \mid M, N\right) \\
n=0,1, \ldots, N-1,
\end{gathered}
$$

to be the probability function of the detector interim output (single measurement) given that $M$ transmit antennas and $N$ receive antennas are used. A similar definition for the AIC detector is achieved by replacing MDL with AIC in (10). It is easily verified that the overall average detection probability is given by

$$
\begin{aligned}
& P_{d}^{M}(L)=L ! \sum_{r_{0} \geq 0, r_{1} \geq 0, \ldots, r_{N-1} \geq 0}\left(\prod_{n=0}^{N-1} \frac{\left(P_{N}^{M}(n)\right)^{r_{n}}}{r_{n} !}\right) \times \\
& \frac{\mathbf{1}_{\left\{r_{M}=\max _{n} r_{n}\right\}}}{\sum_{n=0}^{N-1} \mathbf{1}_{\left\{r_{M}=r_{n}\right\}}},
\end{aligned}
$$

where $\mathbf{1}_{\{\}}$is an indicator function. The probability mass is collected over all the events that the majority decision is correct. In case the majority decision is not unique but still includes the correct answer, the probability mass is divided by the cardinality of the set (reflecting the random selection within this set). Unfortunately, calculating the conditioned probability function $\left\{P_{N}^{M}(n)\right\}$ seems mathematically unfeasible. Therefore, $\left\{P_{N}^{M}(n)\right\}$ are estimated by Monte-Carlo simulations and then substitute into (11) in order to assess the overall probability of detection.

"Soft" combining of measurements is beyond the scope of this paper, and may be considered for further study. A naive combining scheme, which is expected to improve on performance under certain conditions, may be to summarize the AIC/MDL output vector resulting from the $L$ measurements, and then to take the minimum value. i.e. for the MDL we have

$$
\hat{M}=\underset{m=0,1, \ldots, N-1}{\operatorname{argmin}} \frac{1}{L} \sum_{l=1}^{L} \operatorname{MDL}_{l}(m) .
$$

\section{Numerical Results}

In this section Monte-Carlo simulation results $\left(10^{4}\right.$ experiments for each point), demonstrating the impact of various system parameters on the detector performance, are presented. It is noted that all the curves presented in this section are derived for Rayleigh block i.i.d. channels.

The impact of the signal sample size $T$ is demonstrated in Figures 2 and 3 for $M=2$ and $M=3$ respectively. As expected, $P_{d}^{M}(1)$ increases for a given SNR per-receive antenna (SNR $=P /\left(M \sigma^{2}\right)$ ), with the sample size $T$. In addition, the inconsistently of the AIC detector is also observed.

Figures 4 and 5 demonstrate the impact of increasing number of receive antennas $N$ on $P_{d}^{M}(1)$ as a function of

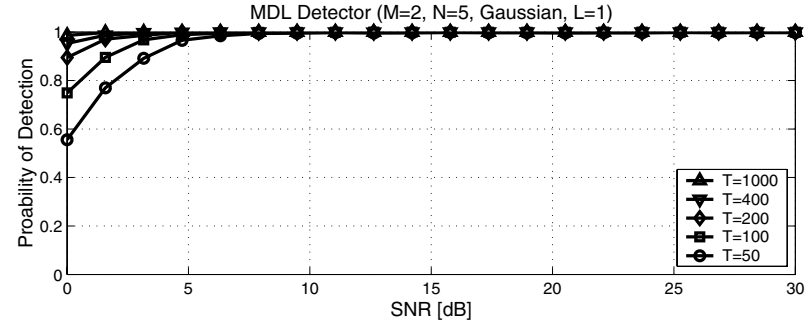

AIC Detector ( $M=2, N=5$, Gaussian, $L=1)$

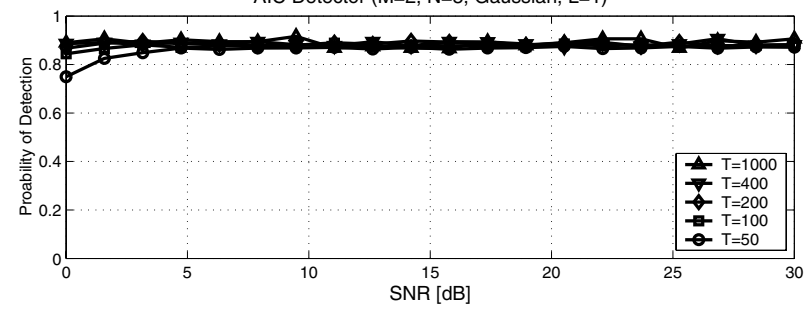

Fig. 2. Detection probability of a single measurement of $T=$ $50,100,200,400,1000$ sample vectors, vs. the SNR per receive antenna for Gaussian input, $M=2$, and $N=5$
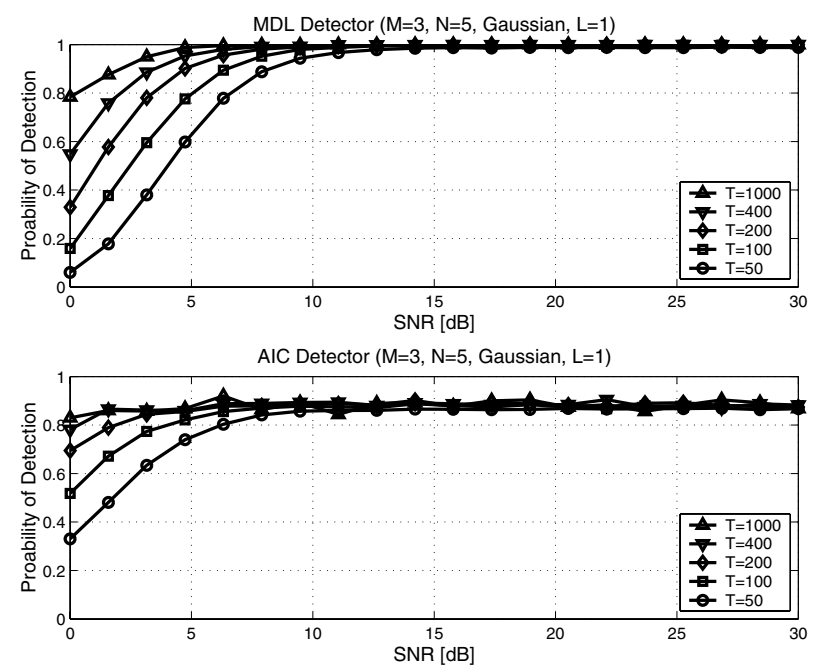

Fig. 3. Detection probability of a single measurement of $T=$ 50, 100, 200, 400, 1000 sample vectors, vs. the SNR per receive antenna for Gaussian input, $M=3$, and $N=5$

the SNR for $M=2$ and $M=3$ respectively. The curves show in general that $P_{d}^{M}(1)$ increases with the number of receive antenna. As an exception to this rule-of-thumb is the AIC curve for $N=M+1$. This phenomenon is explained by the fact that the AIC estimator is inconsistent and it tends to overestimate $M$ [3]. It is evident that when $N=M+1$ the AIC cannot overestimate the number of transmit antennas since it maximal output is $M$ by definition. Therefore, in these cases the AIC estimator is consistent and the probability of detection for a given $T$, increases.

The advantage of having several independent measurements $L$ is demonstrated in Figures 6 and 7 for $M=2, N=6$ and $M=3, N=6$ respectively. As mentioned earlier these curves are derived by substituting the empirical probability 


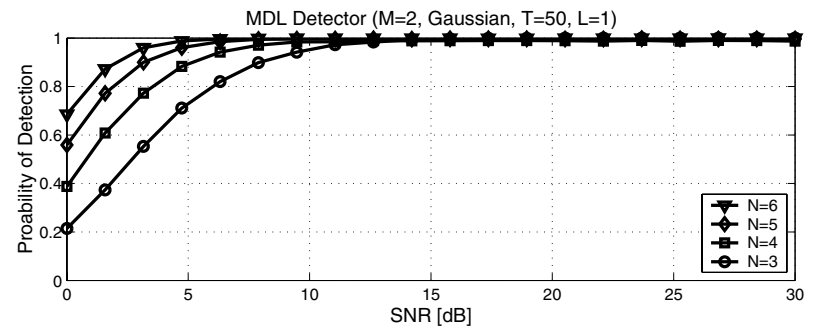

AIC Detector ( $M=2$, Gaussian, $T=50, L=1)$

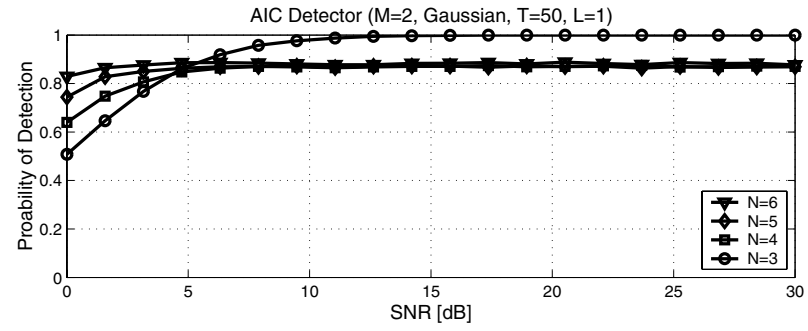

Fig. 4. Detection probability of a single measurement of $T=50$ sample vectors, vs. the SNR per receive antenna for Gaussian input and $M=2$
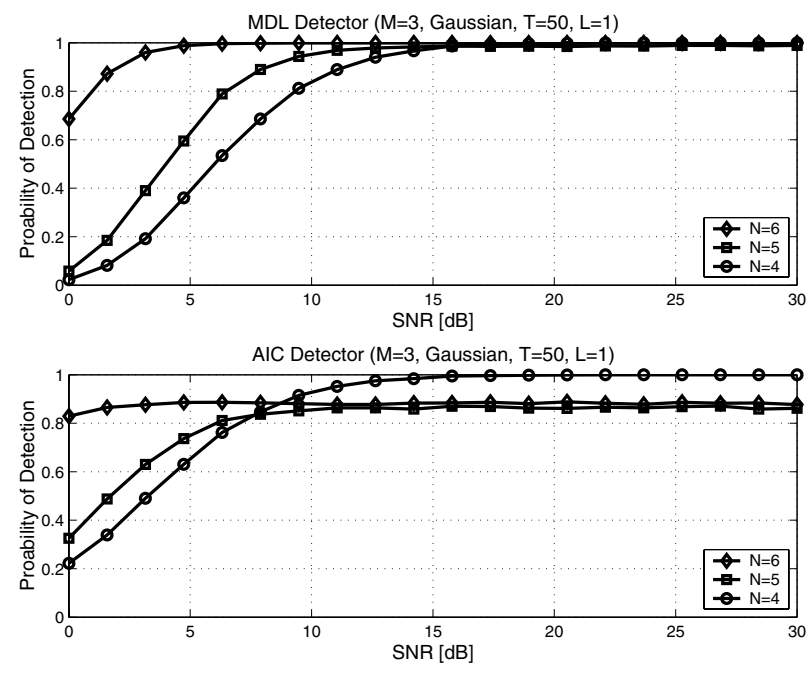

Fig. 5. Detection probability of a single measurement of $T=50$ sample vectors, vs. the SNR per receive antenna for Gaussian input and $M=3$

function $\left\{P_{N}^{M}(n)\right\}$ which was estimated by Monte-Carlo simulation, into (11). It easily verified that in case

$$
P_{N}^{M}(M)>\max _{n=0,1, \ldots(N-1) ; n \neq M} P_{N}^{M}(n),
$$

i.e. the "correct" answer has the largest probability, the overall probability of detection quickly goes to 1 with $L$. On the other hand, if the condition is not satisfied, then particularly for certain SNR range having multiple number of measurements combined in a "hard" manner, reduces the overall probability of detection (see the MDL results presented in Figure 7). It is noted that the condition defines a critical $\mathrm{SNR}_{\mathrm{t}}$ value, for a given system parameter set $(M, N$, and $T)$, which determines whether having multiple number of measurements is beneficial.
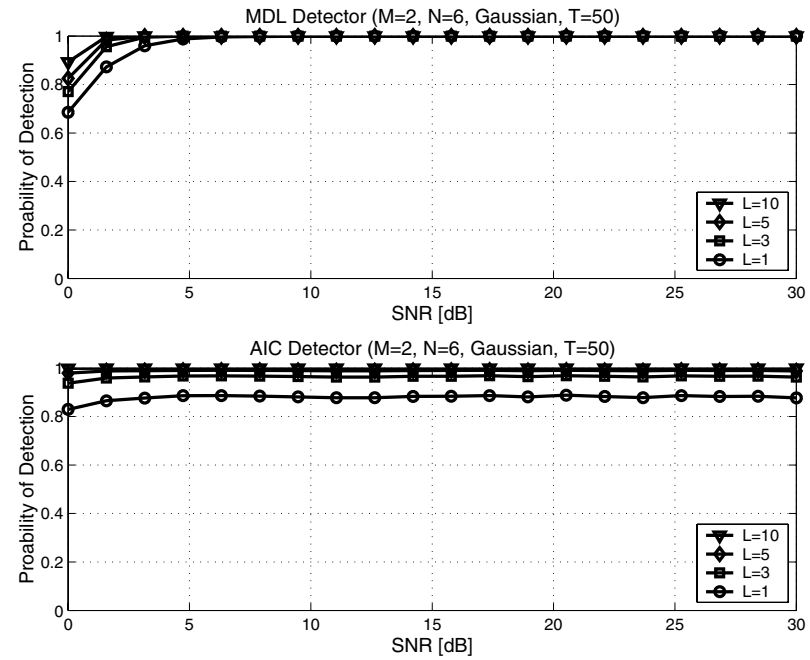

Fig. 6. Detection probability of $L=1,3,5,10$ independent measurements of $T=50$ sample vectors, vs. the SNR per receive antenna for Gaussian input, $M=2$, and $N=6$
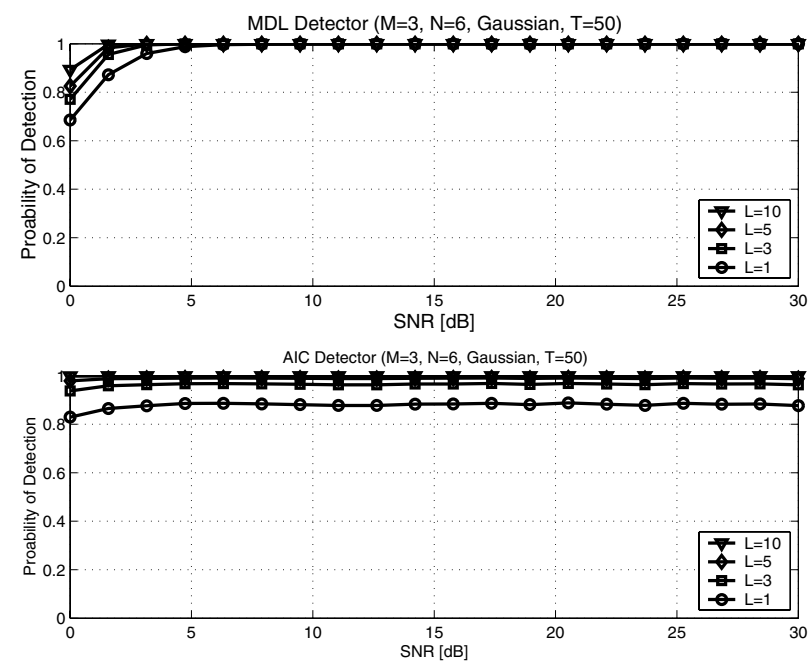

Fig. 7. Detection probability of $L=1,3,5,10$ independent measurements of $T=50$ sample vectors, vs. the SNR per receive antenna for Gaussian input, $M=3$, and $N=6$

\section{Discussion AND FurTher RESEARCH}

In this section we consider several possible extensions to the basic ideal setup presented in Section II. Roughly, the possible directions are divided into three categories: (a) the impact coding, (b) the impact channel characterization, and (c) the impact imperfect interception. It is noted that these directions are currently under investigation. However, some initial thoughts and preliminary results are added in the following.

Finite input alphabet: In Section II the transmitted symbols are assumed to be complex Gaussian random variables. Although Gaussian distribution is the capacity achieving input distribution in cases where the channel is known to the receiver, practical systems use finite alphabet symbols for trans- 


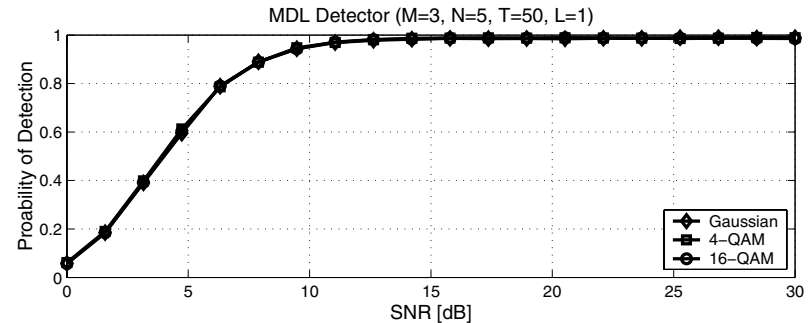

AIC Detector $(M=3, N=5, T=50, L=1)$

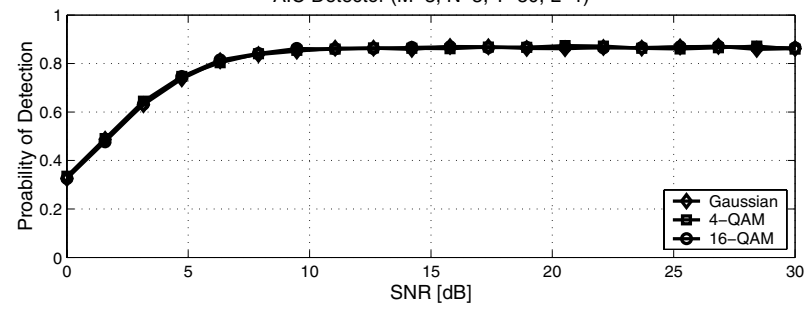

Fig. 8. Detection probability of a single measurement of $T=50$ sample vectors, vs. the SNR per receive antenna for multiplexed Gaussian and QAM input, $M=3$, and $N=5$

mission (e.g. QAM, PSK, etc.). It is noted that developing the optimal AIC/MDL criteria for this case seems mathematically infeasible. A reasonable suboptimal solution may be to use the AIC/MDL criteria developed for the Gaussian input, also for the finite alphabet input. Obviously the criteria are unmatched to the input signal. Nevertheless, preliminary simulations for 4- and 16-QAM rectangular constellations (Figure 8), show that the performance is similar to the performance achieved for Gaussian input (with same average power) over a wide range of the SNR. This result is easily explained by the fact that the AIC/MDL detector is based on the eigenvalues of the sample covariance matrix. Hence, both detectors are based on second order statistics of the input signal. This fact implies that the performance has a weak dependency on the actual input alphabet.

Space-time correlation by coding: In Section II we have assumed that the input symbols feeding each transmitting antenna, are uncorrelated in time and space. Advanced MIMO coding techniques such as beamforming, space-time coding (STC), and dirty-paper coding (DPC) may result in space-time correlation between the input symbols. Using the AIC/MDL estimators is evidently unmatched to these cases and reduced performance is expected since some of the resulting $N-M$ eigenvalues values may be reduced by the induced correlation.

Channel characterization: In Section II we have assumed i.i.d block fading channels. Relaxing this assumption to include Ergodic block fading channels is not expected to change the performance. Another direction is to consider frequency selective fading channels and OFDM systems. It is noted that an extension to frequency selective fading channels is already considered in [3]. Antenna correlation both in the transmit and receive side introduces space correlation and is expected to reduce the performance. Other fading statistics, in addition to the Rayleigh fading channels used in Section V, may be considered as well.

Imperfect interception: In Section II a perfect synchronization is assumed. In practical, phase and frequency error, as well as timing error, are expected to introduce time and space correlation which expected in turn to reduce the performance.

\section{CONCluding Remarks}

This paper provides an initial overlook into the problem of detecting the number of transmit antennas by unauthorized or cognitive terminal. It is shown that under ideal conditions this problem is equivalent to the problem of detecting the number of single antenna transmitters which was treated in [3], where information theoretic criteria are applied to the problem and the MDL/AIC estimators are derived. Numerical results presented for this idealized setup show that the AIC/MDL estimators provides a robust detector for estimating the number of transmit antennas, based on the eigenvalues of the sample covariance matrix $\hat{R}$. Observing the results it is concluded that the MDL is a consistent estimator, while the AIC is a consistent estimator only for $N=M+1$. Since the AIC/MDL use second order statistics, their performance are "insensitive" to the input alphabet as long average power constraints are preserved. The coherence time of the channel (interpreted as the measurement length $T$ ) is a crucial system parameter where for $T \gg N$, the detection probability increases in general with the interceptor reception array size $N$. It is also verified that for a given set of parameters, there is a crucial value of $\mathrm{SNR}_{\mathrm{t}}$, above which "hard" combining of multiple independent measurements quickly brings the final detection probability to 1 . Finally it it is observed that AIC performs better than MDL in the low SNR regime and visa versa. Hence, a combined SNR depended detector is preferable.

As mentioned earlier, several possible extensions to the basic setup may be considered. These extensions render this problem interesting from the theoretical and practical point of views, since detecting the number of transmit antennas is an essential stage in the reception process of both unauthorized and cognitive terminals.

\section{REFERENCES}

[1] E. Telatar, "Capacity of multi-antenna Gaussian channels," European Transactions on Telecommunications, vol. 10, pp. 585-598, Nov. 1999.

[2] G. J. Foschini, "Layered space-time architecture for wireless communication in fading environments when using multi-element antennas," Bell Labs Tech. J., pp. 41-59, 1996.

[3] M. Wax and T. Kailath, "Detection of signals by information theoretic criteria," IEEE transactions on acoustic, speech, and signal processing (ASSP), vol. 33, pp. 387-392, Apr. 1985.

[4] M. S. Bartlett, "A note on the multiplying factors for various $\chi^{2}$ approximations," J. Roy. Stat. SOC., ser. E, vol. 16, pp. 296-298, 1954.

[5] D. N. Lawley, "Tests of significance of the latent roots of the covariance and correlation matrices," Biometrica, vol. 43, pp. 128-136, 1956.

[6] H. Akaike, "Information theory and an extension of the maximum likelihood principle," in Proc. 2nd Int. Symp. Inform. Theory, Suppl. Problems of control and Inform. Theory, pp. 267-281, 1973.

[7] H. Akaike, "A new look at the statistical model identification," IEEE Trans. Automat. Contr., vol. 19, pp. 716-723, 1974.

[8] G. Schwartz, "Estimation the order of a model," Ann. Stat., vol. 6 , pp. 461-464, 1974.

[9] J. Rissanen, "Modeling by shortest data description," Automatica, vol. 14 pp. 465-471, 1978. 